\title{
Detecção de Atividade e Estimação de Canal em Acesso Múltiplo Uplink Grant-Free Usando o Algoritmo BOMP
}

\author{
Ana Luiza Scharf, Bartolomeu F. Uchôa-Filho e Didier Le Ruyet
}

\begin{abstract}
Resumo-Neste trabalho, consideramos o cenário massive Machine-Type Communication (mMTC) baseado no acesso múltiplo Grant-free no qual uma pequena parcela de um número maciço de usuários transmite pequenas quantidades de dados esporadicamente, sem requisitar permissão. $O$ problema de detecção (esparsa) de atividade de usuários (DAU) pode ser resolvido com algoritmos de sensoriamento compressivo (CS), como FOCal Underdetermined System Solver (FOCUSS) e Orthogonal Matching Pursuit (OMP), que foram usados na literatura em canais com desvanecimento plano. Neste trabalho, a partir de um modelo de esparsidade em bloco e fazendo uso de uma aproximação polinomial para a resposta do canal, propomos o uso do algoritmo Block OMP (BOMP) para realizar DAU e estimação do canal (EC) de forma conjunta em canais com desvanecimento seletivo em frequência. Resultados de simulação mostram um excelente desempenho do algoritmo proposto.
\end{abstract}

Palavras-Chave-mMTC, grant-free, detecção de atividade de usuários, estimação de canal, FOCUSS, OMP

Abstract-In this work, we consider the massive Machine-Type Communication (mMTC) scenario based on Grant-free multiple access in which a small portion of a massive number of users transmit small amounts of data sporadically, without requesting permission. The problem of (sparse) user activity detection (DAU) can be solved with compressive sensing (CS) algorithms such as FOCal Underdetermined System Solver (FOCUSS) and Orthogonal Matching Pursuit (OMP), which have been used in the literature for channels with flat fading. In this work, based on a block sparsity model and using a polynomial approximation for the channel response, we propose the use of the Block OMP (BOMP) algorithm to jointly perform DAU and channel estimation (EC) in channels with frequency selective fading. Simulation results show an excellent performance of the proposed algorithm.

Keywords - mMTC, grant-free, user activity detection, channel estimation, FOCUSS, OMP

\section{INTRODUÇÃO}

Em redes de comunicação sem fio convencionais, uma quantidade tipicamente pequena de usuários transmite um grande volume de dados para uma estação base. Nesse cenário, justifica-se perfeitamente o acesso múltiplo ao meio baseado na solicitação e identificação do usuário. Um usuário que deseja enviar dados solicita acesso, que é garantido com alocação

A. L. Scharf e B. F. Uchôa-Filho fazem parte do GPqCom/LCS/EEL, Universidade Federal de Santa Catarina, Florianópolis 88040-900, Brazil (emails: ana.scharf@posgrad.ufsc.br, uchoa@eel.ufsc.br).

D. Le Ruyet faz parte do CEDRIC, Conservatoire National des Arts et Metiers, 75003 Paris, França (e-mail: didier.le ruyet@cnam.fr).

Este trabalho foi parcialmente financiado pelo CNPq (306780/2017-8 e 157663/2019-1) e apoiado pela CAPES através do Programa PrInt CAPESUFSC projeto Automação 4.0. de um recurso ortogonal (tipicamente em frequência ou no tempo). O overhead de controle, gerado nesse processo, é desprezível diante da grande quantidade de dados transmitida e do fato de haver poucos usuários, o que facilita a identificação.

Contrastando com esse cenário, nas futuras redes de comunicação sem fio considera-se também uma grande quantidade de dispositivos (ou máquinas) de IoT (do inglês Internet of Things) [1] conectados. mMTC (do inglês massive MachineType Communication), um dos três cenários contemplados no Sistema $5 \mathrm{G}$, é caracterizado pela existência de um número maciço de dispositivos, com apenas uma pequena parcela deles precisando enviar poucos dados esporadicamente. Essa condição de esparsidade torna oneroso o acesso múltiplo tal qual descrito no parágrafo anterior.

No contexto de mMTC, muitos autores têm considerado o chamado acesso Grant-free [2], i.e., o acesso múltiplo sem solicitação por parte do dispositivo que, por força da tradição, aqui será tratado como usuário. Um usuário que precisa enviar dados o faz sem solicitar permissão. Na outra extremidade, a estação base precisa resolver três problemas. Primeiro, ela precisa identificar quais usuários estão ativos (i,e., estão enviando dados) num determinando momento. Segundo, os canais desses usuários devem ser estimados. Por fim, realizase a detecção dos dados de cada usuário ativo. Neste trabalho, focaremos nos dois primeiros problemas.

Para a detecção de atividade de usuários (DAU), um conjunto finito de sequências (assinaturas) com boas propriedades de correlação é adotado. A qualquer momento, e de forma independente, um usuário que possui dados para transmitir escolhe aleatoriamente uma dessas sequências para servir de sinal piloto. As sequências de Zadoff-Chu (ZC) [3] são frequentemente adotadas nesse contexto [4], [5], [6], pois possuem autocorrelação periódica ideal [7].

Dada a esparsidade intrínseca do sistema, a DAU pode ser vista como um problema de sensoriamento compressivo, cuja solução pode ser obtida por métodos como FOCUSS (do inglês FOCal Underdetermined System Solver) [8] e OMP (do inglês Orthogonal Matching Pursuit) [9]. Cabe mencionar que, pelas suas características, no contexto Grant-free os métodos FOCUSS e OMP se aplicariam de forma direta ao problema de DAU apenas em canais com desvanecimento plano. De fato, uma versão do OMP, DGOMP (do inglês Detection-Based Group OMP), foi utilizada em [10] nesse tipo de canal. Uma estimação de canal (EC) foi também realizada, com a qual a lista de usuários ativos é reduzida.

De forma um tanto quanto forçada, FOCUSS foi adotado 
em [5] para DAU em canais seletivos em frequência usando uma abordagem de aproximação por um canal plano, tendo obtido excelentes resultados. Estendendo o trabalho [5], os autores em [6] realizaram uma EC MMSE (do inglês minimum mean squared error) restrita aos usuários declaradamente ativos, e se valeram de um limiar para refinar essa lista.

Em todas essas propostas, DAU e EC foram realizadas separadamente. Neste trabalho, propomos o método estendido BOMP (do inglês Block OMP) [11] associado a uma aproximação polinomial da resposta em frequência do canal para realizar, de forma conjunta, DAU e EC em canais seletivos em frequência. A proposta será chamada de BOMP-EC. Os receptores existentes citados acima e o receptor proposto são ilustrados na Figura 1.

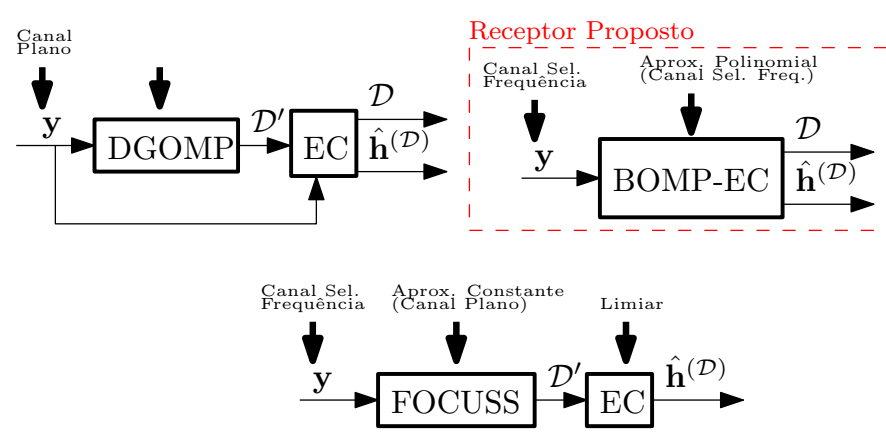

Fig. 1. Receptores considerados neste trabalho.

Este trabalho é organizado da seguinte maneira. Na Secção II, o sistema mMTC é descrito para o cenário de acesso múltiplo Grant-free sob o modelo de canal com desvanecimento seletivo em frequência. Na Secção III, o algoritmo FOCSSUS é apresentado resumidamente. A estimação de canal MMSE com aproximação polinomial é apresentada na Seção IV. O BOMP original é explicado de forma sintética na Seção V. Na seção VI, a modificação do BOMP para realizar a EC conjuntamente com a DAU é apresentada. $\mathrm{Na}$ Seção VII, resultados de simulação são demonstrados. Finalmente, a Seção VIII apresenta as conclusões do trabalho.

Notação: Negrito minúsculo e maiúsculo indicam, respectivamente, vetores e matrizes. Maiúsculo caligráfico representa um conjunto. $(\cdot)^{T}$ denota transposição matricial e $(\cdot)^{H}$, transposição e conjugação matricial. A norma Euclidiana é descrita por $\|\cdot\|_{2}=\sqrt{\mathbf{x}^{H} \mathbf{x}}$; a inversa de Moore-Penrose é definida como $\mathbf{X}^{\dagger}=\mathbf{X}^{H}\left(\mathbf{X X}^{H}\right)^{-1}$. Por fim, $\mathbf{x} \sim \mathcal{C N}(\eta, \mathbf{Z})$ é um vetor aleatório complexo com distribuição Gaussiana com média $\eta$ e matriz de covariância $\mathbf{Z} ; \mathbf{I}_{K}$ denota uma matriz de identidade $K \times K$.

\section{Modelo do Sistema}

O sistema de comunicação uplink em consideração dispõe de $Q$ recursos ortogonais, por exemplo subportadoras de um sistema OFDM (do inglês Orthogonal Frequency-Division Multiplexing), e serve potencialmente $N$ usuários $(N \gg Q)$, dos quais apenas $N_{a}\left(N_{a} \ll N\right)$ estão ativos. Um usuário que deseja transmitir seleciona aleatoriamente uma sequência $\mathrm{s} \in \mathcal{Z} \subset \mathbb{C}^{Q \times 1}$, em que $\mathcal{Z}$ é o conjunto de $|\mathcal{Z}|=U$ sequências piloto e $\mathcal{U}$ representa o conjunto dos índices de $\mathcal{Z}$. Estas são construídas a partir de sequências de ZC [3], dadas por

$$
\left\{\exp \left(-j \frac{\pi r n(n+1+2 q)}{Q^{\prime}}\right)\right\}, n=0, \ldots, Q^{\prime}-1,
$$

em que o comprimento da sequência de $\mathrm{ZC}, Q^{\prime}$, deve ser ímpar e a raiz $r$ e o comprimento $Q^{\prime}$ devem ser relativamente primos. Se $Q^{\prime}$ é escolhido como um número primo, podemos ter $r=$ $1, \ldots, Q^{\prime}-1$. Para cada raiz $r$, podemos ter vários valores de $q \in \mathbb{Z}$, cada um dos quais representa um deslocamento cíclico. Por outro lado, seguindo [4], a DAU apresenta melhor desempenho em um canal com resposta ao impulso de $\tau$ taps se $0 \leq q=q^{\prime} m \leq Q^{\prime}-1$, em que $m \geq \tau$.

$\mathrm{Na}$ prática, é interessante ter as sequências piloto de comprimento par. Assim, neste trabalho, para fins da obtenção da raiz $r$, adotaremos um valor de $Q^{\prime}$ primo, mas adicionaremos um símbolo extra à sequência de ZC. Verificou-se que repetir o primeiro símbolo ao final da sequência produz bons resultados. Com isso, para $Q^{\prime}$ primo, teremos $U=\left(Q^{\prime}-1\right) v$, em que $v=\left\lfloor\frac{Q}{m}\right\rfloor$ e $Q=Q^{\prime}+1$. Temos as seguintes sequências piloto, arranjadas na matriz $\mathbf{S} \in \mathbb{C}^{Q \times U}: \mathbf{S}=\left[\mathbf{s}_{1}, \ldots, \mathbf{s}_{u}, \ldots, \mathbf{s}_{U}\right]$, em que $k=(r-1) v+w, r=1, \ldots, Q^{\prime}-1, w=0, \ldots, v-1$.

$\mathrm{O}$ sinal recebido na estação base, $\mathbf{y} \in \mathbb{C}^{Q \times 1}$, é dado por

$$
\mathbf{y}=\sum_{u \in \mathcal{A}} \operatorname{diag}\left(\mathbf{h}_{u}\right) \mathbf{s}_{u}+\mathbf{n},
$$

em que $\mathcal{A}$ é o conjunto dos índices dos usuários ativos, $\mathcal{A} \subset \mathcal{U}$, $\mathbf{h}_{u} \in \mathbb{C}^{Q \times 1}$ e $\mathbf{s}_{u} \in \mathcal{Z} \subset \mathbb{C}^{Q \times 1}$ representam, respectivamente, os ganhos de canal para os $Q$ recursos e a sequência piloto selecionada aleatoriamente pelo $u$-ésimo usuário ativo (uma coluna de $\mathbf{S})$, e $\mathbf{n} \sim \mathcal{C N}\left(\mathbf{0}, \sigma^{2} \mathbf{I}_{Q}\right)$ indica o ruído complexo aditivo Gaussiano branco.

\section{FOCAL UNDERDETERMINED SySTEM SOLVER}

FOCUSS é um algoritmo iterativo de sensoriamento compressivo que pode ser empregado no contexto de DAU em canais com desvanecimento plano. A partir de $\mathcal{Z}$ e do sinal recebido $\mathbf{y}$, FOCUSS obtém um vetor com $U$ coeficientes que representam as estimativas dos canais vinculados às sequências piloto em $\mathcal{Z}$. Caso uma sequência piloto tenha sido selecionada por um usuário, o coeficiente correspondente será a estimativa do canal deste usuário. Por outro lado, os coeficientes associados a sequencias piloto não selecionadas tendem a apresentar valores próximos de zero. A cada iteração do algoritmo, essa estimativa vai sendo aperfeiçoada. Após a última iteração, os coeficientes são testados em relação a um limiar preestabelecido para fins de detecção de atividade.

FOCUSS é descrito em Algoritmo 1, com as etapas necessárias para realizar a EC conforme as modificações apresentadas em [12]. Cabe destacar que, na versão original do algoritmo [8], os autores indicam um valor inicial para o vetor $\hat{\mathbf{h}}_{1}^{\mathrm{F}}$, mas sugerem que outros podem ser adotados conforme a aplicação, para a obtenção de melhores resultados. Diversos trabalhos apresentam formas de inicialização deste vetor. Em [12], os autores indicam uma possível inicialização do $\hat{\mathbf{h}}_{1}^{\mathrm{F}}$ por MMSE. 


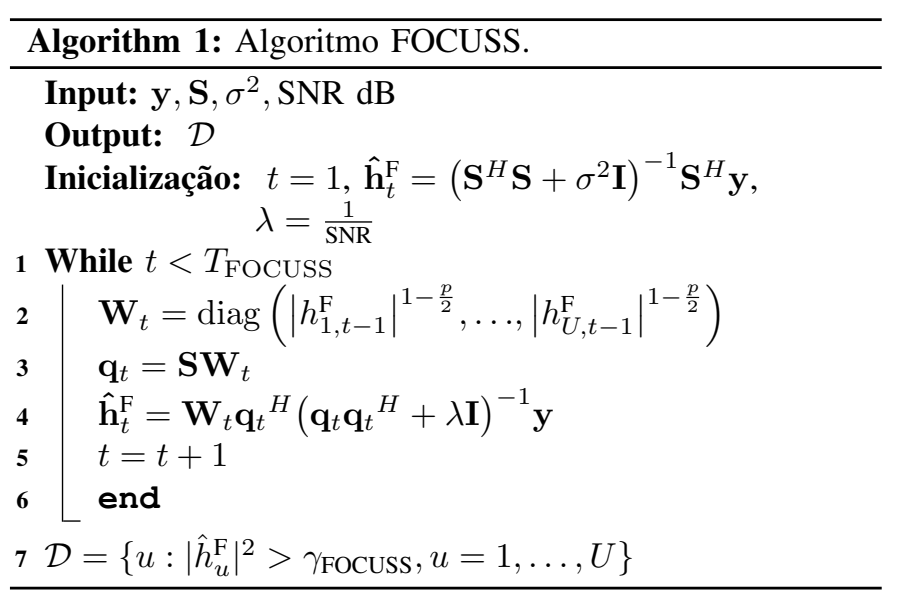

Outras modificações do FOCUSS também foram propostas em [12]. Os autores introduziram o parâmetro $p, p \leq 1$, relacionado a um relaxamento do problema de otimização associado. O valor desse parâmetro que produz o melhor resultado deve ser obtido experimentalmente.

Além do conjunto de índices dos usuários declarados ativos, representado por $\mathcal{D}$, também são definidos os seguintes conjuntos. $\mathcal{C}$ denota o conjunto dos índices dos usuários declarados ativos corretamente, enquanto o conjunto dos falsos ativos é denotado por $\mathcal{F}$. $\log o, \mathcal{D}=\mathcal{C} \cup \mathcal{F}$. O conjunto dos usuários ativos não detectados é denotado por $\mathcal{P}$ (detecção perdida). Assim, o conjunto dos índices dos usuários ativos é $\mathcal{A}=\mathcal{P} \cup \mathcal{C}$. Em um cenário ideal, $\mathcal{D}=\mathcal{C}=\mathcal{A}$ e $\mathcal{P}=\mathcal{F}=\emptyset$. Quanto menor for a cardinalidade dos conjuntos $\mathcal{P}$ e $\mathcal{F}$, melhor será o resultado da DAU.

\section{ESTIMAÇÃO DE CANAL MMSE COM APROXIMAÇÃO POLINOMIAL}

Nesta seção, propomos a EC sob o critério de MMSE com aproximação polinomial do ganho do canal, a ser realizada após a etapa de DAU, feita pelo algoritmo FOCUSS. O vetor de ganho de canal do $u$-ésimo usuário pode ser escrito como

$$
\mathbf{h}_{u}=\sum_{l=0}^{L-1} c_{u}[l]\left(\mathbf{l}^{(l)}\right)+\xi_{u},
$$

em que $\mathbf{l}^{(l)}=\left[0,1,2^{l}, \ldots,(Q-1)^{l}\right]^{T}, \quad \mathbf{c}_{u}=$ $\left[c_{u}[0], \ldots, c_{u}[l], \ldots, c_{u}[L-1]\right]$ são os coeficientes do polinômio de aproximação a serem determinados, $\xi_{u}$ é o vetor que representa o erro da aproximação polinomial e $L$ é o número de coeficientes. Um maior valor de $L$ melhora o resultado da aproximação. Por outro lado, devido à presença de ruído, ter muitos coeficientes a estimar pode representar uma maior fonte de erro de estimação.

Para uma abordagem matricial, precisamos das seguintes definições. Seja $\mathcal{E}=\left\{\epsilon_{1}, \ldots, \epsilon_{e}, \ldots, \epsilon_{E}\right\} \subseteq \mathcal{U}$ algum subconjunto de cardinalidade $|\mathcal{E}|=E \leq U=|\mathcal{U}|$. Definimos o vetor de coeficientes $\mathbf{c}^{\mathcal{E}}=\left[\mathbf{c}_{\epsilon_{1}}^{T}, \ldots, \mathbf{c}_{\epsilon_{e}}^{T}, \ldots, \mathbf{c}_{\epsilon_{E}}^{T}\right]^{T} \in \mathbb{C}^{L E \times 1}$. Também, definimos a matriz de sequências piloto expandida $\mathbf{B}^{\mathcal{E}}=\left[\mathbf{B}_{\epsilon_{1}}^{\mathcal{E}}, \ldots, \mathbf{B}_{\epsilon_{e}}^{\mathcal{E}}, \ldots \mathbf{B}_{\epsilon_{E}}^{\mathcal{E}}\right] \in \mathbb{C}^{Q \times L E}$, em que $\mathbf{B}_{\epsilon_{e}}^{\mathcal{E}}=$ $\left[\mathbf{b}_{\epsilon_{e}}[0], \ldots, \mathbf{b}_{\epsilon_{e}}[l], \ldots, \mathbf{b}_{\epsilon_{e}}[L-1]\right]$. O elemento $\mathbf{b}_{\epsilon_{e}}[l] \in \mathbb{C}^{Q \times 1}$, para $l=0, \ldots, L-1$, é expresso por

$$
\mathbf{b}_{\epsilon_{e}}[l]=\operatorname{diag}\left(\mathbf{l}^{(l)}\right) \mathbf{s}_{\epsilon_{e}},
$$

que pode ser visto como uma nova versão da sequência piloto original, $\mathbf{s}_{\epsilon_{e}}$. Para $l=0$, tem-se que $\mathbf{b}_{\epsilon_{e}}[0]$ é a própria sequência piloto original $\mathbf{s}_{\epsilon_{e}}$.

Com essas definições, podemos rescrever (2) como

$$
\begin{aligned}
\mathbf{y} & =\sum_{u \in \mathcal{A}} \sum_{l=0}^{L-1} c_{u}[l] \operatorname{diag}\left(\mathbf{l}^{(l)}\right) \mathbf{s}_{u}+\mathbf{n}+\xi \\
& =\mathbf{B}^{\mathcal{A}} \mathbf{c}^{\mathcal{A}}+\mathbf{n}+\xi
\end{aligned}
$$

em que $\xi=\sum_{u \in \mathcal{A}} \operatorname{diag}\left(\xi_{u}\right) \mathbf{s}_{u}$ representa o erro da aproximação polinomial dos canais dos usuários em $\mathcal{A}$.

Para $\mathcal{D} \neq \mathcal{A}$, a equação (2) pode ser escrita como

$$
\mathbf{y}=\mathbf{B}^{\mathcal{D}} \mathbf{c}^{\mathcal{D}}+\mathbf{n}+\xi^{\prime}
$$

em que $\xi^{\prime}$ representa o erro de DAU, incluindo os usuários perdidos $(\mathcal{P})$ e os falsos detectados $(\mathcal{F})$, juntamente com erro de estimação devido a aproximação polinomial do canal.

A solução MMSE para os coeficientes $\mathbf{c}^{\mathcal{D}}$ é dada por

$$
\hat{\mathbf{c}}^{\mathcal{D}}=\left(\left(\mathbf{B}^{\mathcal{D}}\right)^{H} \mathbf{B}^{\mathcal{D}}+\sigma^{2} \mathbf{I}\right)^{-1}\left(\mathbf{B}^{\mathcal{D}}\right)^{H} \mathbf{y} .
$$

Deve-se mencionar que a variância utilizada no MMSE da equação (8) foi $\sigma^{2}$, que se refere ao ruído aditivo $\mathbf{n}$. A rigor, esta deveria se referir ao ruído equivalente $\mathbf{n}+\xi^{\prime}$. Apesar dessa simplificação, os resultados apresentados na Seção VII são bastante satisfatórios.

Finalmente, o vetor de ganho de canal estimado do $u$-ésimo usuário, com base na aproximação polinomial, é dado por:

$$
\hat{\mathbf{h}}_{u}=\sum_{l=0}^{L-1} \hat{c}_{u}[l]\left(\mathbf{l}^{(l)}\right) .
$$

\section{DESCRIÇÃO DO BOMP ORIGINAL}

Em [11], os autores consideraram o problema de reconstrução de um vetor $\mathbf{d}$ com esparsidade em bloco, ou seja, o vetor é estruturado em blocos de elementos e apenas um número relativamente pequeno de blocos é sabidamente não nulo. $\mathrm{O}$ algoritmo BOMP proposto em [11] explora essa esparsidade e encontra, iterativamente, o vetor $\mathbf{d}$ a partir da observação

$$
\mathbf{y}^{\prime}=\mathbf{S}^{\prime} \mathbf{d}
$$

em que $\mathbf{d} \in \mathbb{C}^{U \times 1}$ é esparso em bloco e $\mathbf{S}^{\prime} \in \mathbb{C}^{Q \times L U}$ é uma matriz conhecida, chamada de dicionário. É importante destacar que a observação $\mathbf{y}^{\prime}$ em (10) é livre de ruído.

Pela esparsidade em bloco, a matriz $\mathbf{S}^{\prime}$ deve ser dividida em $U$ blocos, na forma

$$
\mathbf{S}^{\prime}=\left[\mathbf{S}^{\prime}[1] \cdots \mathbf{S}^{\prime}[u] \cdots \mathbf{S}^{\prime}[U]\right],
$$

em que cada submatriz $\mathbf{S}^{\prime}[u] \in \mathbb{C}^{Q \times L}$ engloba $L$ colunas.

Sob a hipótese de que as colunas de cada uma das submatrizes $\mathbf{S}^{\prime}[u]$ sejam linearmente independentes, os autores em [11] mostraram que, através da decomposição QR, o sistema em (10) equivale a um sistema no qual cada submatriz é ortonormal. Assim, sem perda de generalidade, pode-se supor que $\mathbf{S}^{\prime}[u], u=1, \ldots, U$, tenham esta propriedade. 
$\mathrm{O}$ algoritmo inicia com um vetor residual $\mathbf{r}_{1}=\mathbf{y}^{\prime}$ e o conjunto dos usuários detectados $\mathcal{D}=\emptyset$. A cada iteração $t>$ 1 , um novo índice $u_{t}$ é adicionado a $\mathcal{D}$. Este índice é associado ao subespaço $\mathbf{S}^{\prime}[u]$ com máximo casamento em relação ao resíduo da iteração anterior:

$$
u_{t}=\underset{u \in\{\mathcal{U}-\mathcal{D}\}}{\arg \max }\left\|\left(\mathbf{S}^{\prime}[u]\right)^{H} \mathbf{r}_{t-1}\right\|_{2} .
$$

O conjunto das soluções temporárias é obtido por

$$
\left\{\mathbf{d}_{t}[u]\right\}_{u \in \mathcal{D}}=\arg \min _{\left\{\mathbf{x}[u] \in \mathbb{C}^{L \times 1}\right\}_{u \in \mathcal{D}}}\left\|\mathbf{y}^{\prime}-\sum_{u \in \mathcal{D}} \mathbf{S}^{\prime}[u] \mathbf{x}[u]\right\|_{2}^{2}
$$

Por fim, atualiza-se o resíduo:

$$
\mathbf{r}_{t}=\mathbf{y}^{\prime}-\sum_{u \in \mathcal{D}} \mathbf{S}^{\prime}[u] \mathbf{d}_{t}[u] .
$$

O número de iterações do BOMP está vinculado ao grau de esparsidade do vetor $\mathbf{d}$. Após encontrar todos os blocos não nulos, o algoritmo finaliza suas iterações.

Para um cenário com ruído e/ou com esparsidade desconhecida, porém com $U=1$, i.e., o algoritmo OMP, os autores em [13] propuseram o seguinte critério de parada, que pode facilmente ser estendido para o BOMP: finalize as iterações e assuma que encontrou todos os blocos não nulos quando

$$
\left\|\mathbf{r}_{t}\right\|_{2} \geq \sigma \sqrt{Q+2 \sqrt{Q \log Q}}
$$

em que $\sigma$ é o desvio padrão do ruído.

\section{Algoritmo PROposto: BOMP-EC}

Nesta seção, aplicamos o algoritmo BOMP para a detecção de atividade de usuários e estimação de canal conjuntas no sistema descrito na Seção II, que considera desvanecimento seletivo em frequência. Além disso, usaremos a aproximação polinomial para o canal, conforme apresentado na Seção IV.

$\mathrm{O}$ algoritmo proposto utiliza $\mathbf{B}^{\mathcal{U}} \in \mathbb{C}^{1 \times L U}$ como dicionário, cada bloco do qual associado a um usuário/sequência piloto. $\mathrm{A}$ cada iteração, o BOMP-EC escolhe um novo usuário para incluir ao conjunto $\mathcal{D}$ e recalcula os coeficientes de aproximação polinomial de todos usuários contidos em $\mathcal{D}$.

O Algoritmo 2 mostra todas as operações realizadas no BOMP proposto para encontrar todos os usuários ativos e calcular os respectivos coeficientes. A minimização na equação (13) é realizada no Passo 5 do Algoritmo 2, em que $\mathbf{d}_{t}[u]$ é equivalente ao nosso $\mathbf{c}^{\mathcal{D}}$. Além disso, a cada iteração os valores dos vetores $\mathbf{c}^{\mathcal{D}}$ dos usuários declarados ativos são atualizados para alcançar uma maior precisão. Ao final do algoritmo BOMP-EC, os vetores de ganho de canal estimado dos usuários contidos em $\mathcal{D}$ são obtidos via equação (9).

\section{RESUlTAdos NumÉRICOS}

Nas simulações, assumimos $\mathcal{A}=6$ usuários ativos. Foram utilizados dois comprimentos de sequências piloto: $Q=24$ $\left(Q^{\prime}=23\right)$ e $Q=48\left(Q^{\prime}=47\right)$. O modelo de canal seletivo em frequência adotado foi o EPA (do inglês "Extended Pedestrian A model") com uma frequência de Doppler de $5 \mathrm{~Hz}$

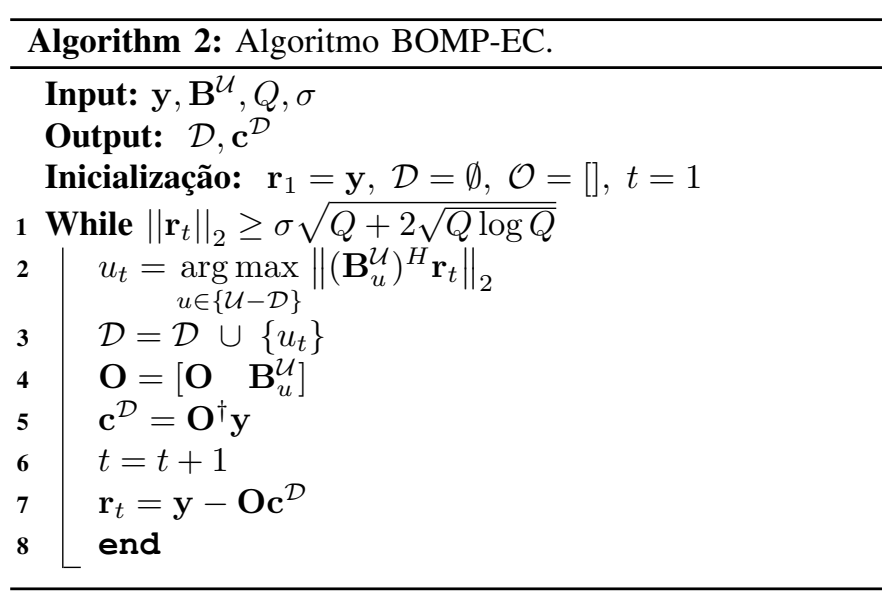

com 4 taps [14], o que nos fez adotar $m=4$. Assim, obtemos $U=132$ e $U=552$, respectivamente.

Um total de 1000 iterações por SNR (do inglês "Signal-toNoise Ratio") foram realizadas. Seguindo a literatura [5], [6], adotamos $T_{\text {FOCUsS }}=20$ e $\gamma_{\text {FOCUSS }}=0.01$. Para o Passo 2 do Algoritmo 1, verificou-se que $p=0.8$ trouxe os melhores resultados para esta configuração. A aproximação polinomial da Seção IV foi realizada com $L=2$ e $L=3$.

A detecção de atividade de usuário foi avaliada em relação à métrica $F_{1}$, dada por

$$
\begin{aligned}
F_{1} & =\frac{2 \times \text { Precisão } \times \text { Sensibilidade }}{\text { Precisão }+ \text { Sensibilidade }} \\
& =\frac{2|\mathcal{C}|}{2|\mathcal{C}|+|\mathcal{F}|+|\mathcal{P}|},
\end{aligned}
$$

em que precisão e sensibilidade são definidas como:

$$
\text { Precisão }=\frac{|\mathcal{C}|}{|\mathcal{C}|+|\mathcal{F}|}, \quad \text { Sensibilidade }=\frac{|\mathcal{C}|}{|\mathcal{C}|+|\mathcal{P}|} .
$$

Esta métrica representa o desempenho da DAU levando-se

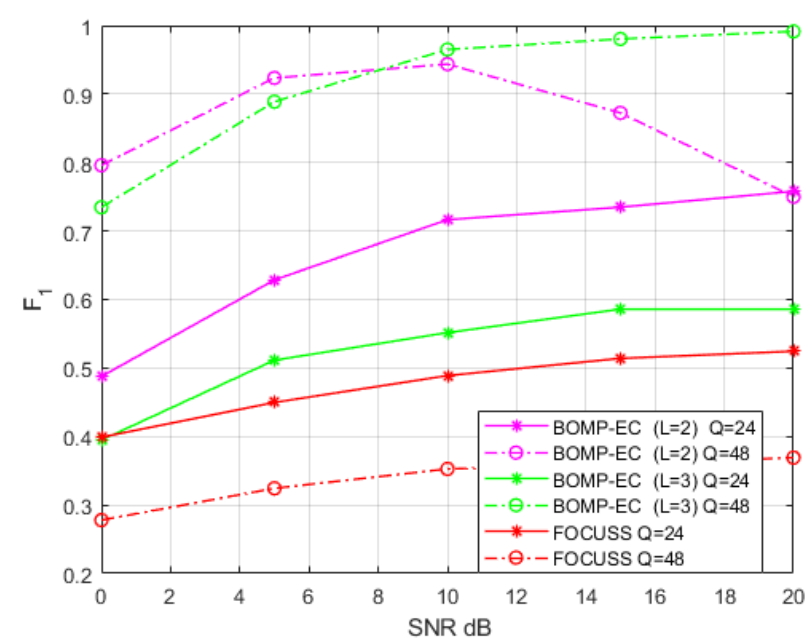

Fig. 2. Métrica $F_{1}$ para os algoritmos FOCUSS e BOMP-EC (proposto).

em consideração os falsos ativos e as detecções perdidas, 
com mesmo peso. Quanto mais próxima de 1 (valor máximo), melhor é o desempenho.

A Figura 2 apresenta os resultados da métrica $F_{1}$ para os algoritmos FOCUSS e proposto BOMP-EC. Percebe-se que o comprimento da sequência piloto tem um grande impacto no desempenho do BOMP-EC. Em geral, quanto maior este comprimento, melhor o desempenho. Esse comportamento é ainda mais acentuado quando o parâmetro $L$ da aproximação polinomial é maior. Outro resultado observado é que $L$ deve ser suficientemente grande para que a métrica $F_{1}$ do BOMPEC se aproxime de 1 à medida que a SNR aumenta. Uma exceção é o caso observado para $Q=48$ e $L=2$, em que há, na verdade, uma perda de desempenho em altas SNRs. Isso pode estar associado ao critério de parada (15), desenvolvido originalmente para o OMP, que para o BOMP pode precisar de um refinamento. Na Figura 2, como o FOCUSS não realiza a EC do canal seletivo em frequência, o valor de $L$ não é considerado para este algoritmo.

O segundo parâmetro de desempenho avaliado foi o MSE (do inglês "Mean Square Error") entre os ganhos dos canais estimados e os ganhos dos canais verdadeiros dos usuários declarados ativos corretamente, ou seja, os usuários de $\mathcal{C}$. A Figura 3 apresenta os resultados de MSE contemplando os mesmos algoritmos e os mesmos parâmetros apresentados na Figura 2 para a métrica $F_{1}$. É perceptível que a estimação MMSE do FOCUSS para o canal seletivo em frequência não apresenta resultados adequados. O problema não é necessariamente o método de estimação de canal em si, mas uma consequência da presença de usuários falsos ativos e detecções perdidas, que influenciam negativamente na estimação de canal do FOCUSS. Como o FOCUSS teve os piores resultados na métrica $F_{1}$, os resultados negativos de MSE eram esperados.

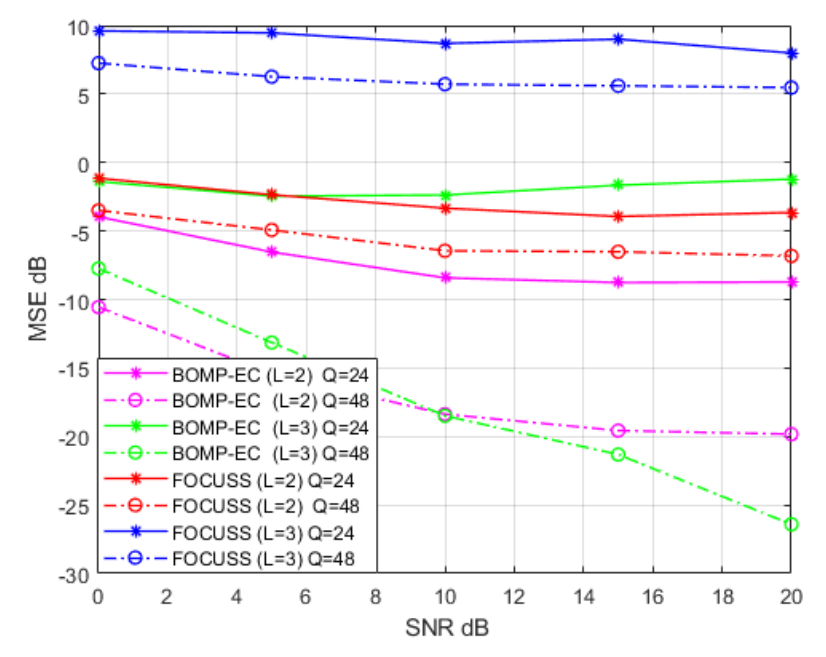

Fig. 3. MSE em relação ao conjunto $\mathcal{C}$.

Com os resultados acima pode-se concluir que o algoritmo de DAU e EC conjuntas proposto, BOMP-EC, é um excelente candidato para um cenário de múltiplo acesso em sistemas Grant-free. De modo geral, a escolha dos parâmetros do BOMP-EC depende de fatores como: canal, comprimento da sequência piloto, número de usuários ativos e o grau do polinômio para estimação.

\section{CONCLUSÃO}

Neste trabalho, propusemos uma modificação no algoritmo BOMP para realizar detecção de atividade de usuários e estimação de canal conjuntamente em um sistema de acesso múltiplo Grant-free sob o modelo de canal com desvanecimento seletivo em frequência. $\mathrm{O}$ algoritmo FOCUSS da literatura foi usado como referência para a etapa de detecção de atividade. Uma estimação de canal MMSE com aproximação polinomial, em relação aos usuários declarados ativos pelo FOCUSS, foi proposta. Desse modo, este algoritmo também pôde ser usado na comparação da estimação de canal. Resultados de simulação revelaram um excelente desempenho do algoritmo proposto BOMP-EC em relação a ambas as funções. Sendo assim, a contribuição deste trabalho é de grande interesse para o cenário mMTC das redes sem fio de última geração.

\section{REFERÊNCIAS}

[1] C. Bockelmann, N. Pratas, H. Nikopour, K. Au, T. Svensson, C. Stefanovic, P. Popovski, and A. Dekorsy, "Massive machine-type communications in 5g: physical and mac-layer solutions," IEEE Communications Magazine, vol. 54, no. 9, pp. 59-65, 2016.

[2] M. B. Shahab, R. Abbas, M. Shirvanimoghaddam, and S. J. Johnson, "Grant-free non-orthogonal multiple access for iot: A survey," IEEE Communications Surveys Tutorials, vol. 22, no. 3, pp. 1805-1838, 2020.

[3] D. Chu, "Polyphase codes with good periodic correlation properties (corresp.)," IEEE Trans. on Information Theory, vol. 18, no. 4, pp. 531$532,1972$.

[4] A. T. Abebe and C. G. Kang, "Grant-free uplink transmission with multicodebook-based sparse code multiple access (mc-scma)," IEEE Access, vol. 7, pp. 169853-169864, 2019.

[5] A. Bayesteh, E. Yi, H. Nikopour, and H. Baligh, "Blind detection of scma for uplink grant-free multiple-access," in 2014 11th International Symposium on Wireless Communications Systems (ISWCS), 2014, pp. 853-857.

[6] F. Wang, Y. Zhang, H. Zhao, H. Huang, and J. Li, "Active user detection of uplink grant-free scma in frequency selective channel," in 2018 IEEE 87th Vehicular Technology Conference (VTC Spring). IEEE, 2018, pp. $1-6$.

[7] M. Hua, M. Wang, K. W. Yang, and K. J. Zou, "Analysis of the frequency offset effect on zadoff-chu sequence timing performance," IEEE Trans. on Communications, vol. 62, no. 11, pp. 4024-4039, 2014.

[8] I. F. Gorodnitsky and B. D. Rao, "Sparse signal reconstruction from limited data using focuss: A re-weighted minimum norm algorithm," IEEE Trans. on Signal Processing, vol. 45, no. 3, pp. 600-616, 1997.

[9] J. Tropp, A. C. Gilbert et al., "Signal recovery from partial information via orthogonal matching pursuit," IEEE Trans. Inform. Theory, vol. 53, no. 12 , pp. 4655-4666, 2007.

[10] J. Liu, G. Wu, S. Li, and O. Tirkkonen, "Blind detection of uplink grant-free scma with unknown user sparsity," in 2017 IEEE International Conference on Communications (ICC). IEEE, 2017, pp. 1-6.

[11] Y. C. Eldar, P. Kuppinger, and H. Bolcskei, "Block-sparse signals: Uncertainty relations and efficient recovery," IEEE Trans. on Signal Processing, vol. 58, no. 6, pp. 3042-3054, 2010.

[12] S. Bhat and A. Chockalingam, "Sparsity-exploiting detection of largescale multiuser gsm-mimo signals using focuss," in 2016 IEEE 83rd Vehicular Technology Conference (VTC Spring), 2016, pp. 1-5.

[13] T. T. Cai and L. Wang, "Orthogonal matching pursuit for sparse signal recovery with noise," IEEE Trans. on Information Theory, vol. 57, no. 7, pp. 4680-4688, 2011.

[14] 3GPP, "Evolved Universal Terrestrial Radio Access (E-UTRA); Radio Resource Control (RRC); Protocol specification," 3rd Generation Partnership Project (3GPP), Technical Specification (TS) 36.331, 042017 , version 14.2.2. 